\title{
Breakup of filaments in blends during simple shear flow
}

\author{
Peter Van Puyvelde, Hong Yang, ${ }^{\text {a) }}$ Jan Mewis, and Paula Moldenaers ${ }^{\text {b) }}$ \\ Department of Chemical Engineering, Katholieke Universiteit Leuven, \\ de Croylaan 46, B-3001 Heverlee, Belgium
}

(Received 25 April 2000; final revision received 18 July 2000)

\begin{abstract}
Synopsis
In this paper the breakup of droplets under shear in polymer blends is studied by means of linear conservative dichroism and small angle light scattering. More specifically breakup of long fibrils by interfacial instabilities is considered. Measurements are performed on dilute model systems containing nearly Newtonian components in transient flows that involve a sudden increase or decrease in shear rate. The experimental results are used to evaluate the Khakhar and Ottino theory [Khakhar and Ottino (1987)]. In this theory breakup times are calculated starting from the onset of the instability. It is demonstrated that the scaling derived from the Khakhar and Ottino theory also holds for the more readily accessible total breakup time, calculated from the onset of shear flow in a startup flow. The development of interfacial disturbances is studied in a flow history, which consists of generating fibrils by suddenly applying a shear rate followed by a sudden drop in shear rate during which the breakup process is observed. The evolution of the dominant wavelength during this process turns out to be time controlled rather than strain controlled as suggested by the theory. (C) 2000 The Society of Rheology. [S0148-6055(00)00706-9]
\end{abstract}

\section{INTRODUCTION}

When flow is applied to an emulsion or a liquid polymer blend, the droplets of the dispersed phase will deform and possibly break or coalesce. Droplet deformation is promoted by the viscous stress $\eta_{m} \dot{\gamma}$ (with $\eta_{m}$ the matrix viscosity and $\dot{\gamma}$ the shear rate) generated by the external flow and counteracted by the interfacial stress $\Gamma / R$ (with $\Gamma$ the interfacial tension and $R$ the radius of the droplets) [Taylor (1934)]. The relative magnitude of the competing stresses can be expressed by the capillary number $(\mathrm{Ca}$ $=\eta_{m} \dot{\gamma} R / \Gamma$ ). Low values of Ca result in stable, deformed droplets [Grace (1982); Bentley and Leal (1986)]. When the viscous stress dominates the interfacial stress, i.e., with $\mathrm{Ca}$ larger than a critical value $\mathrm{Ca}_{c}$, the deformed droplets will become unstable and break [Acrivos (1983); Rallison (1984)].

Breakup of droplets during flow does not happen instantaneously. When the viscosity ratio $p$ is in the range of $0.1-1.0$, droplets first deform into slender filaments [Rumscheidt and Mason (1961)]. While the droplets are being continuously stretched, small disturbances that are present on the surface of the filaments start to grow and eventually cause the filaments to disintegrate. For a filament in a quiescent matrix, a dominant disturbance

\footnotetext{
${ }^{a}$ Present address: Department of Chemical Engineering, University of California, Santa Barbara, Santa Barbara, CA 93106.

${ }^{b)}$ Author to whom correspondence should be addressed. Electronic mail:

paula.moldenaers@cit.kuleuven.ac.be; phone: +32-16-322359; fax: +32-16-322991.
} 
with a specific wavelength has the fastest growth rate and consequently causes the rupture [Tomotika (1935)]. This phenomenon can be considered an interfacial variety of the Rayleigh instabilities at free surfaces. In a flowing system, the interfacial disturbances are damped by the external flow, thus delaying the breakup until the droplets are more deformed [Mikami et al. (1975); Janssen and Meijer (1993)].

By assuming that the disturbances grow in an axisymmetric way, Tomotika (1936) and later Mikami et al. (1975) studied theoretically the breakup of an elongated Newtonian droplet in a Newtonian matrix during extensional flow. Khakhar and Ottino (1987) extended the analysis to general linear flows. They took into account a small nonaxisymmetric phase shift of the disturbances caused by the flow near the surface of the filaments. The Khakhar and Ottino theory has been experimentally verified by Janssen and Meijer (1993) with Newtonian fluids in planar extensional flow for both breakup time and resulting droplet size. Up to now, no experimental evaluation of the theory seems to have been performed under simple shear flow conditions.

Investigating filament breakup during flow requires a measurement technique which can track the development of the disturbances. Rheo-optical techniques provide the means to meet this need. The time evolution of the linear conservative dichroism in particular can be used to identify deformation, breakup, and coalescence of droplets subjected to shear flows [Yang et al. (1998); Vermant et al. (1998); Van Puyvelde et al. (1998)]. It has also been demonstrated that breakup of elongated filaments induced by interfacial instabilities causes a characteristic multiple-streak small angle scattering (SALS) pattern, at least in a quiescent matrix [Mewis et al. (1998); Van Puyvelde et al. (1998)]. In the present work, linear conservative dichroism as well as SALS will be used to study the effect of shear flow on the breakup of filaments.

\section{THE KHAKHAR AND OTTINO THEORY}

Khakhar and Ottino (1987) considered the deformation and breakup of a liquid cylinder with a small axisymmetric, periodic disturbance on its surface immersed in an immiscible matrix and subjected to a general linear flow. Under these conditions the velocity field, with respect to a frame rotating with the cylinder, is essentially an axisymmetric extensional flow on which a nonaxisymmetric shear flow along the surface of the cylinder is superimposed. The extensional flow component stretches the filament and simultaneously increases the wavelength of any existing disturbance. The shear flow component causes the initially axisymmetric disturbance to become nonaxisymmetric.

Assuming the deformation of the filament due to the surface instability to be small and the disturbances only marginally asymmetric, Khakhar and Ottino (1987) derived expressions for the changes of the average radius $r$ of the filament, the wavelength $\lambda_{R}$ and the amplitude $\alpha$ of the disturbance. These are based on a perturbation analysis of the disturbed flow around the filament. The rates of change for $r, \lambda_{R}$, and $\alpha$ at a given shear rate are given by

$$
\begin{gathered}
\dot{r}=-\frac{G_{1}(t) r}{2}, \\
\dot{k}_{R}=-G_{1}(t) k_{R}, \\
\dot{\alpha}=-\frac{G_{1}(t) \alpha}{2}+k_{R}\left[A_{1} I_{1}\left(k_{R} r\right)+A_{2} r I_{1}^{\prime}\left(k_{R} r\right)\right],
\end{gathered}
$$


where $k_{R}=2 \pi / \lambda_{R}$ is the wave number; $A_{1}, A_{2}$ are complex expressions that can be found in the literature [Mikami et al. (1975)]; $I_{1}\left(k_{R} r\right)$ is a modified Bessel function of the first order, $I_{1}^{\prime}\left(k_{R} r\right)$ its derivative with respect to $r$. The stretching rate $G_{1}$ is given by

$$
G_{1}(t)=\frac{\dot{\gamma}\left(\cot \vartheta_{0}+\dot{\gamma} t\right)}{1+\left(\cot \vartheta_{0}+\dot{\gamma} t\right)^{2}},
$$

where $\vartheta_{0}$ is the angle between the long axis of the filament and the velocity of the simple shear flow at the moment the disturbances start to grow (at time $t_{0}$ ).

Defining a dimensionless wave number $x=k_{R} r$, and using the initial values $x_{0}$ $=k_{R, 0} r_{0}$ and $\alpha_{0}$, Eq. (3) can be integrated [Khakhar and Ottino (1987)]

$$
\ln \frac{\alpha}{\alpha_{0}}=\int_{x}^{x_{0}}\left[\frac{x_{0}}{6 \tan \vartheta_{0} E} \frac{\left(1-x^{2}\right)}{x^{2}} \phi(x)-\frac{(p-1)}{x} \bar{\phi}(x)\right] d x+\frac{1}{3} \ln \frac{x}{x_{0}},
$$

where $E$ is $\dot{\gamma} \eta_{m} r_{0} /(\sqrt{2} \Gamma)$, with $r_{0}$ the initial radius of the filament and $\phi(x)$ and $\bar{\phi}(x)$ known functions of the dimensionless wave number [e.g., Mikami et al. (1975)]. The initial conditions $r_{0}, \alpha_{0}$, and $\vartheta_{0}$ correspond to the instant at which a stretched filament just becomes unstable with respect to surface disturbances, i.e., the moment at which the fatal disturbance starts to grow. Kuhn (1953) proposed an estimate for $\alpha_{0}$ based on thermal fluctuations: $\sqrt{21 k_{B} T /\left(8 \pi^{3 / 2} \Gamma\right)}$, with $k_{B}$ Boltzmann's constant and $T$ the absolute temperature. This prediction yields a value of order $10^{-3} \mu \mathrm{m}$ for $\alpha_{0}$. On the basis of rheological experiments Vinckier et al. (1997) recently reported that the limiting value for the initial amplitude at low concentrations of the dispersed phase becomes similar to the Kuhn or thermal value for the filament breakup in a quiescent matrix.

The evolution of the average radius $r$ can be calculated from Eqs. (1) and (4) to be $r=r_{0}\left(1+2 \tan \vartheta_{0} \gamma\right)^{-1 / 2}$, with $\gamma$ the applied strain. Khakhar and Ottino compared the evolution of $r(t)$ with that of $\alpha(t)$, according to Eq. (5). For disturbances with a small initial wave number the amplitude of the disturbance is always smaller than the filament radius, so no breakup occurs. Only when the disturbance has a large enough initial wave number, can it grow sufficiently large to break the filament under flow. Among these the smallest wave number will cause the actual breakup as the larger ones grow slower.

\section{MATERIALS AND METHODS}

The components used in this study are a poly(butadiene) (PB) from Janssen Chimica, two types of poly(isobutene) (PIB) from Exxon Chemical, and two types of poly(dimethylsiloxane) (PDMS) from Rhône-Poulenc. The steady state rheological properties $\left(23^{\circ} \mathrm{C}\right)$ of the components have been measured on a Rheometrics mechanical spectrometer RMS800, using cone and plate geometry (see Fig. 1). The viscosity of the pure components is nearly constant over the shear rate range covered. The high molecular weight PDMS (Rhodorsil 47V200000, longest relaxation time $0.027 \mathrm{~s}$ ) is the most elastic component, although the measured normal stresses are still quite small. For the high molecular weight of PIB (Parapol 1300) the normal stresses are even smaller, whereas for the PB and the low molecular weight PIB (Parapol 950) no significant normal stresses could be detected at all. As the polymers are liquid at room temperature, problems with thermal degradation, which are often encountered in melts, are avoided. Four model blends have been used in this study. The polymer combinations, with information on viscosity ratio and interfacial tension are given in Table I. The interfacial tension has been measured by a new rheo-optical technique [Van Puyvelde et al. (1998)]. The con- 


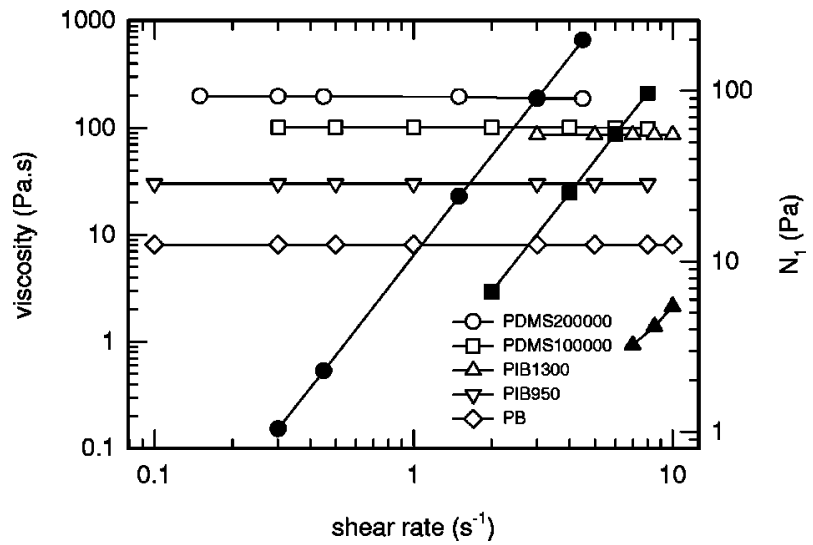

FIG. 1. Rheological characteristics of the components at $23{ }^{\circ} \mathrm{C}$ (open symbols: viscosity, closed symbols: $\mathrm{N}_{1}$ ).

centration of the blends is kept low (typically $1 \%$ or $3 \%$ ) in order to ensure sufficient transparency for optical measurements.

Linear conservative dichroism measurements have been conducted on a rheo-optical analyzer (ROA) (Rheometric Scientific) using parallel plate geometry. Light from a $\mathrm{He}-\mathrm{Ne}$ laser (wavelength $\lambda=0.633 \mu \mathrm{m}$ ) first passes through a modulator that consists of a polarizer with its polarization direction perpendicular to the flow direction and a half-wave plate rotating at $2 \mathrm{kHz}$. The modulated light then propagates in the velocity gradient direction through the flow cell. After leaving the sample, the light is directly collected on a detector at zero scattering angle. The resulting signal is sent to two lock-in amplifiers to provide the in-phase and out-of-phase components from which the dichroism can be calculated [Fuller (1995)]. The rheo-optical method uses a local measurement; i.e., the laser beam always passes the sample at the same distance with respect to the center of the cell. Hence, the shear rate that is probed is always the same during an experiment. SALS measurements have been performed on a modified ROA device, the detector being replaced by a semitransparent sheet. The scattering pattern is recorded with a charge coupled device camera (Ikegami model ICD-810P) and then either sent to a monitor for direct observation or digitized by a PC equipped with a Data Translations DT3851 frame grabber for image analysis. The maximum accessible scattering angle is $15^{\circ}$ in air.

TABLE I. Blend systems.

\begin{tabular}{|c|c|c|c|c|c|c|}
\hline Blend & Minor phase & Matrix phase & $\begin{array}{l}\text { Viscosity } \\
\text { ratio } p\end{array}$ & $\underset{(\mathrm{mN} / \mathrm{m})}{\Gamma}$ & $\begin{array}{c}\dot{\gamma}_{s} \\
\left(\mathrm{~s}^{-1}\right)\end{array}$ & $\begin{array}{c}R \\
(\mu m)\end{array}$ \\
\hline 1 & $\begin{array}{c}1 \% \text { PDMS } \\
\text { (Rhodorsil 47V200000) }\end{array}$ & $\begin{array}{c}\text { PIB } \\
\text { (Parapol 1300) }\end{array}$ & 2.3 & 4.7 & 2 & 2.53 \\
\hline 2 & $\begin{array}{c}1 \% \text { PIB } \\
\text { (Parapol 1300) }\end{array}$ & $\begin{array}{c}\text { PDMS } \\
\text { (Rhodorsil 47V200000) }\end{array}$ & 0.44 & 3.0 & 2 & 2.35 \\
\hline 3 & $\begin{array}{c}3 \% \mathrm{~PB} \\
(\mathrm{Mw}=4500)\end{array}$ & $\begin{array}{c}\text { PIB } \\
\text { (Parapol 950) }\end{array}$ & 0.27 & 0.2 & 1 & 2.53 \\
\hline 4 & $\begin{array}{c}\text { 1\% PDMS } \\
\text { (Rhodorsil 47V100000) }\end{array}$ & $\begin{array}{c}\text { PIB } \\
\text { (Parapol 1300) }\end{array}$ & 1.1 & 3.1 & $\begin{array}{l}3 \\
1\end{array}$ & $\begin{array}{l}1.1 \\
3.2\end{array}$ \\
\hline
\end{tabular}




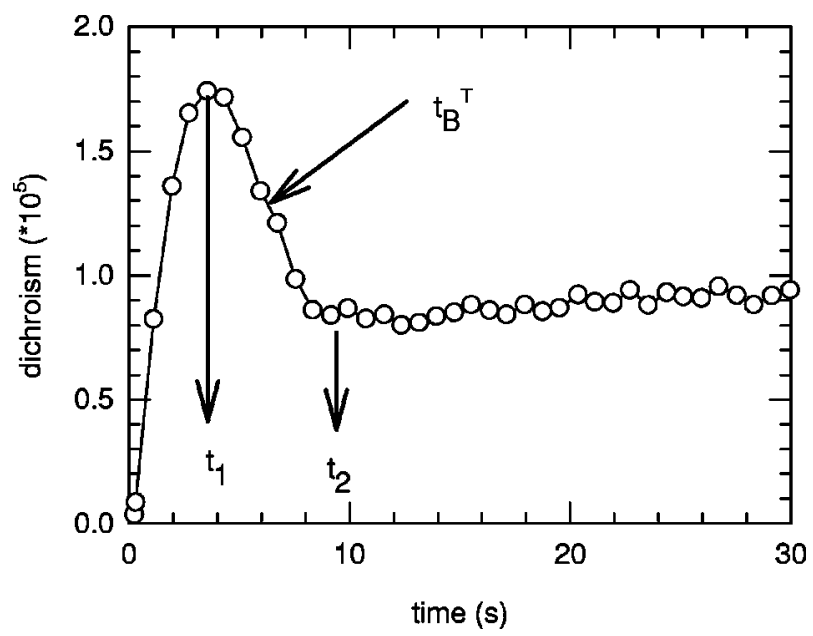

FIG. 2. Dichroism evolution for a stepup in shear rate from 2 to $30 \mathrm{~s}^{-1}$ (blend 1).

In immiscible polymer blends the droplet size is governed by the shear history. In the present experiments, a reproducible droplet size has been achieved by shearing at a given preshear rate $\left(\dot{\gamma}_{s}\right.$ in Table I for 5000 strain units) until the dichroism reached a steady state value. It should be pointed out that reaching a constant response does not necessarily imply that the resulting structure is independent of the previous shear histories. At low shear rates hysteresis phenomena may be encountered [Minale et al. (1997)]. It has however been verified that this is not the case here. The mean droplet radii (see $R$ in Table I) produced by the preshearing operation have been calculated from the SALS patterns by means of the Debye-Bueche method [Yang et al. (1998)]. The scattering method only gives an average droplet size. No direct information on the width of the droplet size distribution can be deduced from this type of measurement. However, microscopy data on similar systems have shown that the droplet size distribution after a preshearing operation is fairly monodisperse [Grizzuti and Bifulco (1997)].

\section{RESULTS AND DISCUSSION}

\section{A. Determination of breakup time during stepup flows}

The breakup process under shear is studied here by means of a shear history that consists of two parts. During a preshearing period a controlled initial morphology is generated (see above). Subsequently the shear rate is suddenly increased and the evolution of dichroism and SALS is monitored. Figure 2 shows a typical time evolution of the dichroism during such a stepup in shear rate for a rather large step ratio, i.e., from 2 to 30 $\mathrm{s}^{-1}$. The increase in shear rate causes the droplets to stretch, and the resulting rise in shape anisotropy entails a growing dichroism. While the droplets are continuously stretched, their cross section becomes smaller and consequently the interfacial stress increases. As a result interfacial instabilities develop that eventually lead to disintegration of the filament. During this process, the anisotropy and hence the dichroism decreases. At still longer times, the dichroism is seen to increase slightly again. This can be attributed to coalescence as discussed earlier [Yang et al. (1998)].

Although the instant at which the filament finally ruptures can be situated in the decreasing part of the dichroism curve (Fig. 2), the exact moment cannot be identified on the curve. Recently, the total breakup time $t_{B}^{T}$ of droplets, starting from their undeformed 


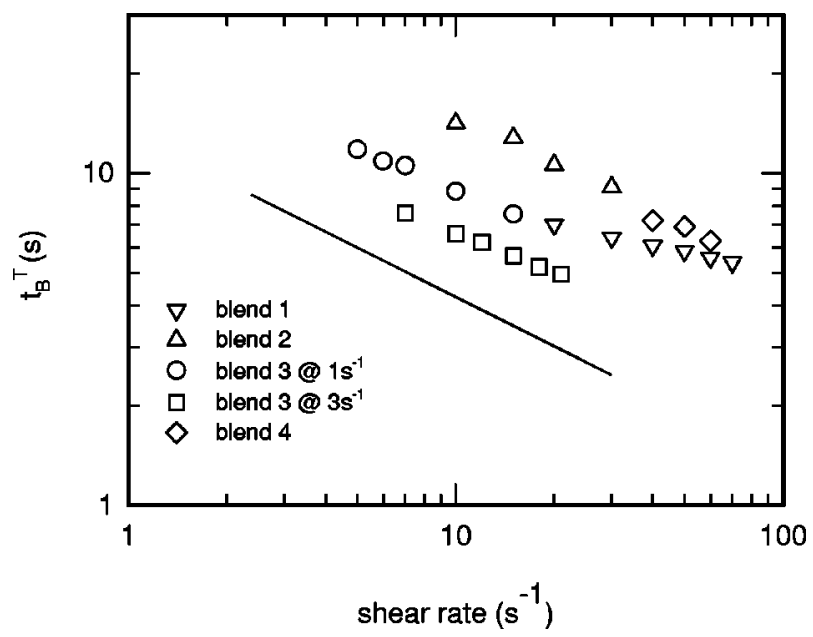

FIG. 3. Total breakup times as a function of shear rate for the different blend systems investigated in this work. Initial conditions as specified in Table I. The full line corresponds to a slope of $-1 / 3$ and is added to guide the eye.

state, could be determined more accurately by measuring the dichroism and its orientation angle in the velocity-gradient plane, using a Couette cell [Vermant et al. (1998)]. Before breakup, the filaments are nearly oriented in the velocity direction. After breakup, smaller droplets are formed which, at equilibrium, should make a larger angle with the velocity direction. Hence the moment at which the orientation angle starts to move away from the flow direction signals the breakup of the stretched filaments. This is found to happen close to the time halfway between the times corresponding to the maximum ( $t_{1}$ in Fig. 2) and the minimum ( $t_{2}$ in Fig. 2) values of the dichroism. Experiments in a Couette cell could only be performed with the rather transparent PB/PIB blend. The blends consisting of PIB and PDMS are too turbid to be probed in this geometry. To avoid an arbitrary choice for the breakup time, the halfway point between the maximum and minimum values of the dichroism, measured in a parallel plate geometry, has been used to determine the total breakup time $t_{B}^{T}$ (see Fig. 2). Figure 3 shows $t_{B}^{T}$ as a function of shear rate for the different blends investigated here. The initial droplet sizes $R$ for each blend are given in Table I.

\section{B. Breakup time after a sudden increase in shear rate}

The breakup time $t_{B}^{T}$ includes the deformation stage as well as the actual breakup stage. The Khakhar and Ottino theory however only covers the growth process of the surface disturbances. In order to compare the experimental results of Fig. 3 with the theory, it is necessary to know the time at which the fatal disturbance starts to grow. There is no straightforward manner to determine this time in a flowing system. The disturbances can be expected to start growing once the interfacial stresses overcompensate the viscous ones. For a highly stretched droplet, the interfacial stress far from the ends of the droplet equals $\Gamma / r$. The viscous stress may be approximated by $G_{1}(t) \eta_{m}$ [Khakhar and Ottino (1987)]. A balance consequently implies

$$
G_{1}\left(t_{0}\right) \eta_{m} \approx \frac{\Gamma}{r_{0}}
$$




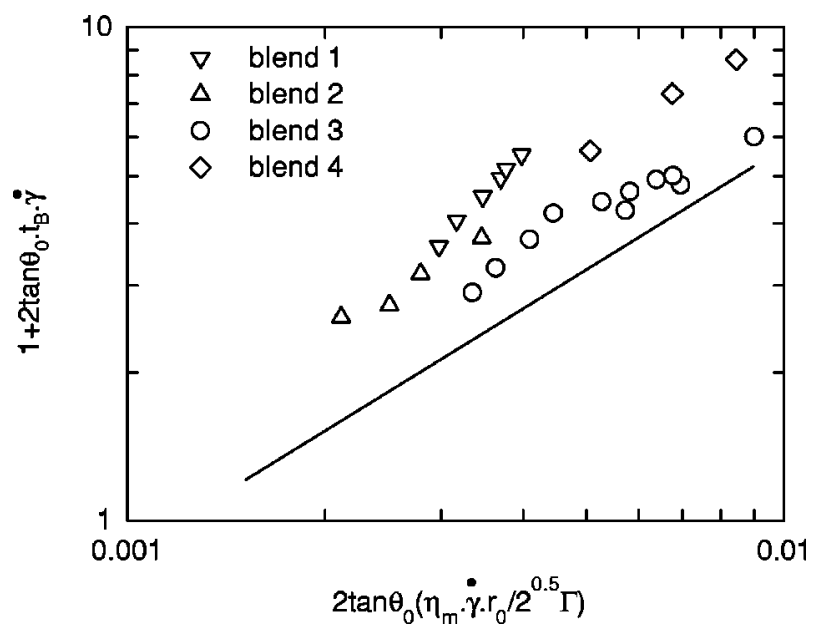

FIG. 4. Scaling relation proposed by Khakhar and Ottino [Eq. (9)] for the breakup time as a function of shear rate. The full line is to guide the eye and has a slope of 0.65 .

with $r_{0}$ the radius of the fibril at $t_{0}$, the time at which disturbances start to grow. Assuming affine deformation and large strains one finds [Elemans et al. (1993)]

$$
\begin{aligned}
& r_{0} \approx R\left(\dot{\gamma} t_{0}\right)^{-1 / 2}, \\
& \tan \vartheta_{0} \approx 1 /\left(\dot{\gamma} t_{0}\right) .
\end{aligned}
$$

Hence Eq. (4) becomes $G_{1}\left(t_{0}\right) \approx 1 /\left(2 t_{0}\right)$. This would lead to the following expression for $t_{0}$ :

$$
t_{0} \approx\left(\frac{R \eta_{m}}{2 \Gamma}\right)^{2 / 3} \dot{\gamma}^{-1 / 3}
$$

In the experiments the maximum of the dichroism ( $t=t_{1}$ in Fig. 2$)$ signals the onset of a substantial growth of the interfacial disturbance. This moment can be estimated to be closely related to time $t_{0}$ of the theory. The measured values of $t_{1}$ have been verified to be proportional to $\dot{\gamma}^{-1 / 3}$, a scaling suggested by Eq. (8) to hold for $t_{0}$.

For filament breakup in a simple shear flow, Khakhar and Ottino (1987) derived numerically a nondimensional relation to estimate the breakup time $t_{B}$

$$
1+2 \tan \vartheta_{0} t_{B} \dot{\gamma} \div\left(2 \tan \vartheta_{0} \frac{\eta_{m} \dot{\gamma} r_{0}}{\sqrt{2} \Gamma}\right)^{\mu},
$$

with $\mu=0.65$, independent of the viscosity ratio $p$. When using $t_{1}$ to estimate $t_{0}$, values for $r_{0}$ and $\vartheta_{0}$ can be calculated assuming affine deformation between $t=0$ and $t_{1}$. In Fig. 4 the scaling relation of Eq. (9) is evaluated. As can be seen the slope of 0.65 (solid line) agrees reasonably well with the experimental results. Although the scaling is qualitatively followed, no absolute comparison with the theory can be made, lacking the well-defined initial conditions required by the Khakhar and Ottino theory. 


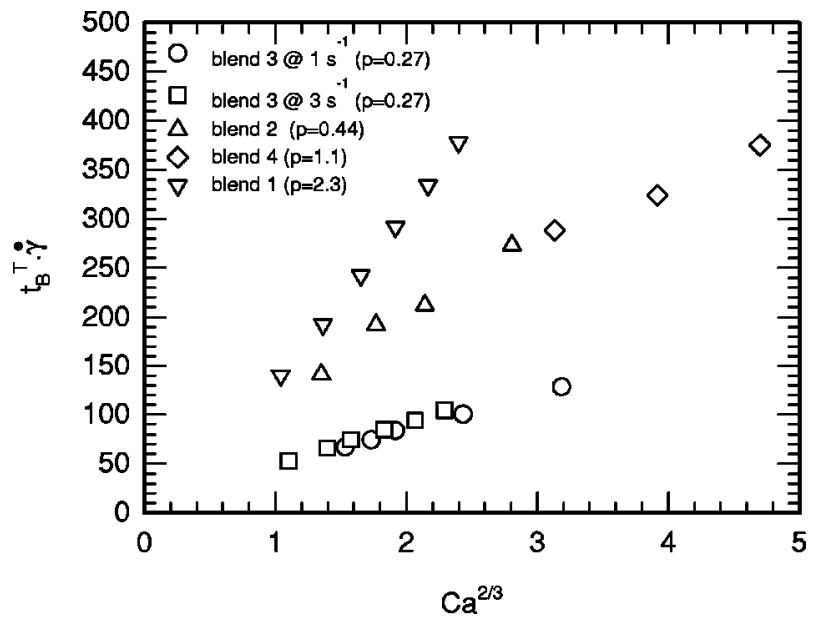

FIG. 5. Dimensionless breakup time as a function of $\mathrm{Ca}^{2 / 3}$ for different blend systems.

For practical purposes it is more convenient to use the total breakup time $t_{B}^{T}$ rather than $t_{B}$ as defined in the Khakhar and Ottino theory. Assuming affine deformation in order to estimate $r_{0}$ and $\vartheta_{0}$ [Eq. (7)] and for large enough strains at rupture, Eq. (9) becomes

$$
t_{B} \div \frac{(2 \sqrt{2})^{\mu}}{2}\left(\frac{R \eta_{m}}{2 \Gamma}\right)^{2 / 3} \dot{\gamma}^{-1 / 3}
$$

Comparing Eqs. (8) and (10), it can be seen that both $t_{0}$ and $t_{B}$ depend on $R \eta_{m} / \Gamma$ and $\dot{\gamma}$ in a similar fashion. Hence the same dependence should also hold for $t_{B}^{T}$

$$
t_{B}^{T} \div\left[\left(\frac{R \eta_{m}}{2 \Gamma}\right)^{2 / 3}\right] \dot{\gamma}^{-1 / 3} .
$$

Equation (11) suggests that the total breakup time, starting from an undeformed droplet of radius $R$, is proportional to $\dot{\gamma}^{-1 / 3}$. The experimental results displayed in Fig. 3 follow this relation quite well even for values of $t_{B}^{T} \dot{\gamma}$ that are too small to satisfy Eq. (10). In addition, the agreement between theory and experiment is remarkably good considering that it is a linearized theory and strictly only valid for small deformations of the cylindrical filament. Only the slope of blend 1 seems to deviate systematically. The total breakup times for this blend, which consists of 1\% PDMS (Rhodorsil V200.000) in PIB (Parapol 1300), are proportional to $\dot{\gamma}^{-0.22}$ rather than to $\dot{\gamma}^{-1 / 3}$. This blend is, however, the one that contains slightly elastic droplets. As pointed out by various authors [e.g., Van Oene (1972)] elasticity tends to stabilize droplets. A similar trend is found here: elasticity of the droplet phase acts to stabilize the dispersed phase, leading to a slower decrease of breakup time with shear rate.

Equation (10) can be rewritten to express a dimensionless breakup time

$$
t_{B}^{T} \dot{\gamma} \div \mathrm{Ca}^{2 / 3} \text {. }
$$

This expression contains the radius of the undeformed droplet, an experimentally accessible characteristic. In Fig. 5 the dimensionless total breakup times of Fig. 3 are shown as 


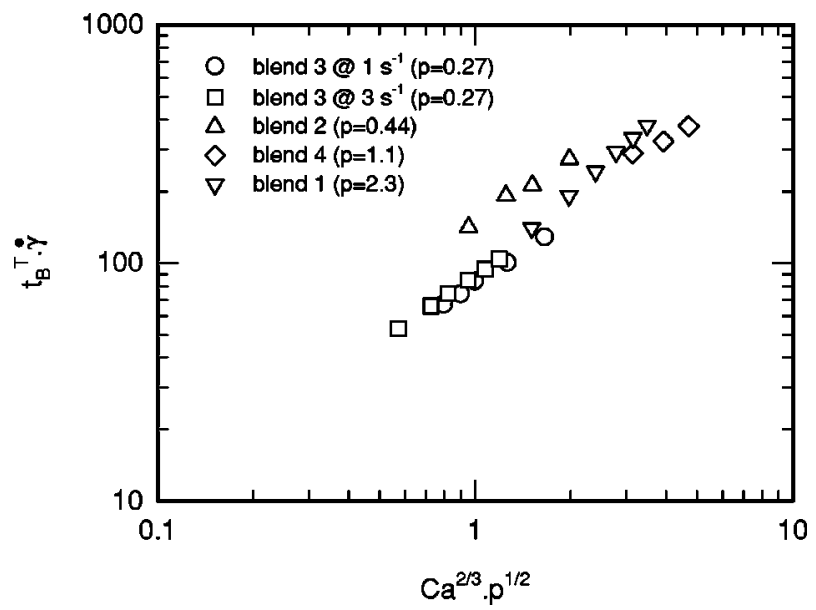

FIG. 6. Scaling of the dimensionless breakup time: empirical reduction for viscosity ratio $p$.

a function of $\mathrm{Ca}^{2 / 3}$. Quasilinear relations are obtained for the different blends. The scaling of Eq. (12) also superimposes experiments on blend 3 with different preshearing rates and hence with different initial droplet sizes. It can be concluded that the scaling proposed by Khakhar and Ottino for $t_{B}$ can be applied to the total time for breakup, starting from undeformed droplets.

The suggested scaling for $t_{B}^{T}$ does not reduce data for different blends to a single curve. This can be achieved with an empirical scaling factor based on the viscosity ratio $p$ (Fig. 6). As the viscosity has been varied between 0.27 and 2.3 , the proposed scaling covers the relevant range of viscosity ratios for the case of polymer blends. Perhaps surprisingly, the experimental breakup points for the blend containing the elastic droplets can also be superimposed. It should be pointed out, however, that the elasticity has already been taken into account to a certain extent for this blend. Indeed, according to Table I, the interfacial tension of blend 1 is $4.7 \mathrm{mN} / \mathrm{m}$. In principle this value should be the same as for the reverse blend $(3 \mathrm{mN} / \mathrm{m})$. However, the rheo-optical method used to determine the interfacial tension measures an "effective" interfacial tension [Van Puyvelde et al. (1998)]. Since droplet elasticity enhances the "effective" interfacial tension and hence stabilizes the droplets, this method produces a higher value for the interfacial tension. Blend 2 does not obey the proposed scaling relation of Fig. 6. It contains a viscoelastic matrix, this apparently has to be taken into account explicitly.

\section{Breakup of filaments after a sudden reduction in shear rate}

As discussed above dichroism measurements can be used to extract quantitative information about the breakup times during stepup flows. Whether a kind of Rayleigh instability is responsible for the droplet breakup however cannot be deduced unambiguously from these experiments. For highly stretched fibrils under quiescent conditions, growing instabilities at the interface give rise to multiple-streak SALS patterns [Mewis et al. (1998)]. Analysis of this pattern can yield information about the degree of dispersion in the blend. Although this feature was also expected to be present during flow, it was not observed in simple stepup transients. In order to study the development of interfacial disturbances during shear a different flow history has been used.

After a preshearing period to generate a well defined droplet size, the shear rate is suddenly increased for a short time to create long filaments. Subsequently the shear rate 


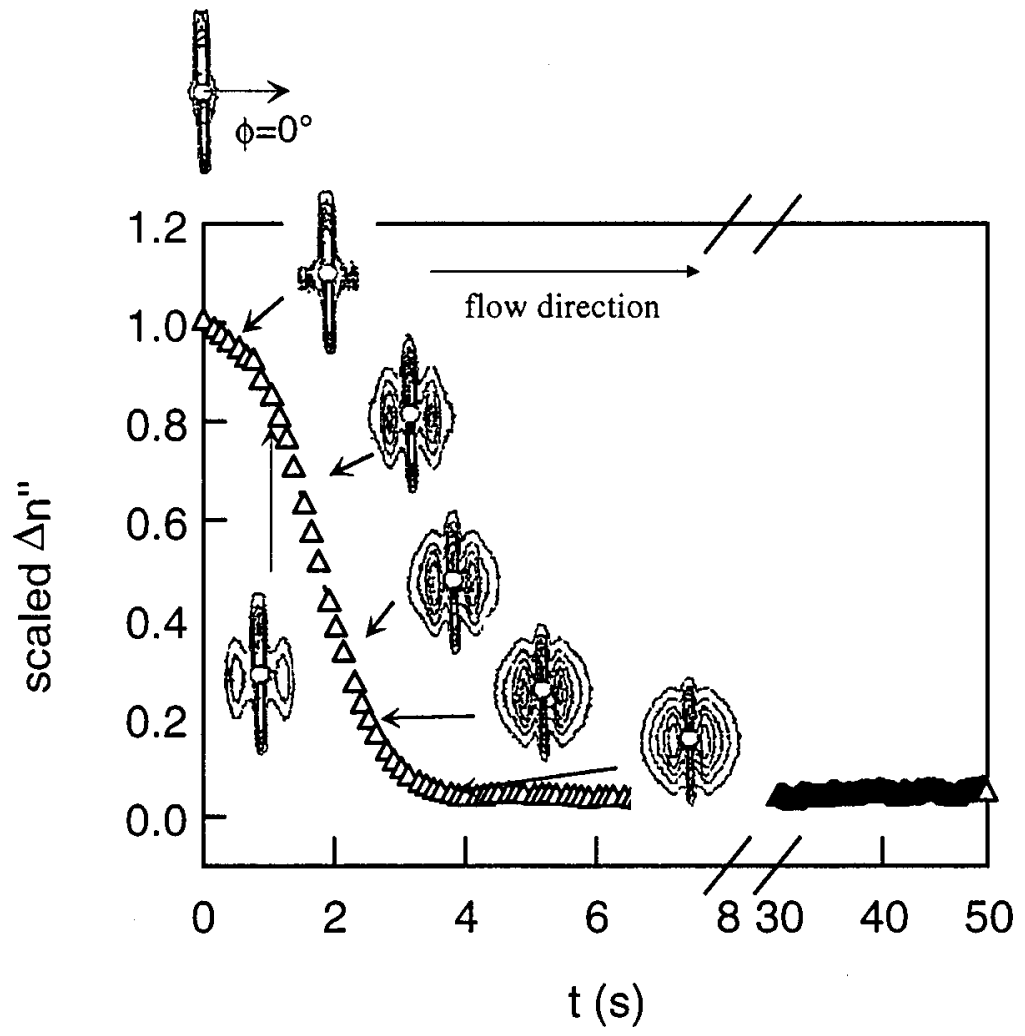

FIG. 7. Time evolution of the scaled dichroism and corresponding SALS patterns in a stepdown experiment after shearing for $3 \mathrm{~s}$ at $30 \mathrm{~s}^{-1}$ and then stepping down to $5 \mathrm{~s}^{-1}$ (blend 4).

is decreased again and the breakup of the filaments is observed. Shearing at the higher shear rate should be limited to times shorter than the time required to reach the maximum in dichroism in order to avoid premature breakup of the filaments. Here the filaments (blend 4) have been generated by shearing at $30 \mathrm{~s}^{-1}$ for $3 \mathrm{~s}$, which in this case corresponds to a capillary number nearly seven times the critical value. This ensures affine deformation of the droplets and produces long slender filaments as required by the Khakhar and Ottino theory [Khakhar and Ottino (1987)]. For a stepup flow at such a shear rate, the time to reach the maximum dichroism is about $4 \mathrm{~s}$.

For blend 4 sheared for $3 \mathrm{~s}$ at $30 \mathrm{~s}^{-1}$, the length, radius and orientation angle of the deformed droplets with respect to flow direction have been calculated to be $576 \mu \mathrm{m}, 0.34$ $\mu \mathrm{m}$, and $0.6^{\circ}$ respectively. From the rheo-optical data a value of $0.41 \mu \mathrm{m}$ was derived for the radius of the filaments [Mewis et al. (1998)] which is close to the one calculated from the affine deformation theory. When arresting the flow at that moment, the characteristic multiple-streak SALS pattern for filament breakup via Rayleigh instabilities can be seen to develop [Mewis et al. (1998)]. When the flow is suddenly slowed down rather than being arrested, the rheo-optical response still remains qualitatively similar as illustrated in Fig. 7. It can be seen that immediately after slowing down the shear rate a secondary peak starts to develop indicating that, at the moment the shear rate is changed, disturbances are already present on the interface.

Figure 8 shows the dichroism evolution after stepdown experiments for different final shear rates starting from the same initial fibrillar structure. As the final shear rate ap- 


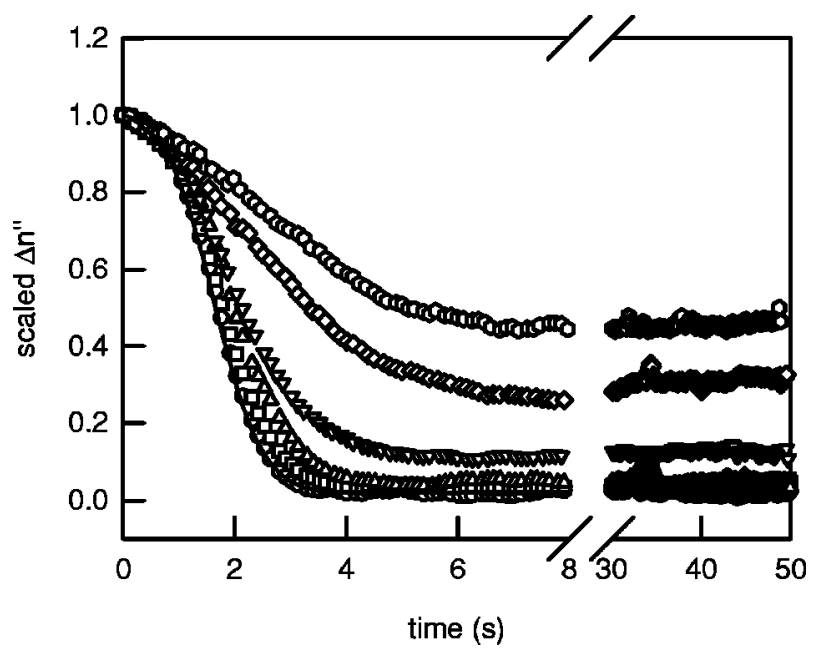

FIG. 8. Time evolution of the scaled dichroism for different stepdown experiments after shearing for $3 \mathrm{~s}$ at 30 $\mathrm{s}^{-1}$ (blend 4). The final shear rates are: $(-) 0 \mathrm{~s}^{-1}$, (o) $2 \mathrm{~s}^{-1},(\square) 5 \mathrm{~s}^{-1},(\triangle) 8 \mathrm{~s}^{-1},(\nabla) 10 \mathrm{~s}^{-1},(\diamond) 15 \mathrm{~s}^{-1}$, (口) $20 \mathrm{~s}^{-1}$.

proaches the one at which the droplets have been stretched previously, the dichroism curve loses its shoulder and the multiple-streak SALS patterns become less distinct (not shown). These changes might be attributed to an interference of the shear flow with the development of the interfacial instabilities. Indeed the shearing motion leads to a distortion of the axisymmetric disturbances and therefore also to a reduction of their rheooptical signature.

As long as the dichroism curve displays the specific shoulder for Rayleigh instabilities, the breakup time of the fibrils $t_{B}$ can be estimated as in a quiescent matrix [Van Puyvelde et al. (1998)]. This procedure consists of plotting the slope of the dichroism versus time; its minimum is attributed to the breakup time. The Khakhar and Ottino analysis can be applied to the present shear history since the initially formed slender fibrils are still subjected to a shear flow. The experimental results are compared with the theoretical predictions in Fig. 9. Here the time at which the shear rate is suddenly decreased has been assumed to coincide with the onset of the growth of the fatal disturbance. At very low shear rates no breakup times could be obtained from the theory as the calculations to solve Eq. (3) diverged. At higher shear rates a slight increase of the breakup time with shear rate is predicted which is confirmed by the experiments. The absolute values of $t_{B}$ seem to be in reasonable agreement with the theory in this region. In stepdown flows the effect of shear on the breakup time turns out to be limited in the range of shear rates covered.

When the filaments break via Rayleigh instabilities in a quiescent matrix, the scattering angle of the secondary streaks $\theta_{m}$ is known to remain constant [Mewis et al. (1998)]. This scattering angle is related to the wavelength of the dominant disturbance by [Mewis et al. (1998)]

$$
\theta_{m}=\arcsin \left(\frac{\lambda_{l}}{n_{m} \lambda_{R}}\right),
$$




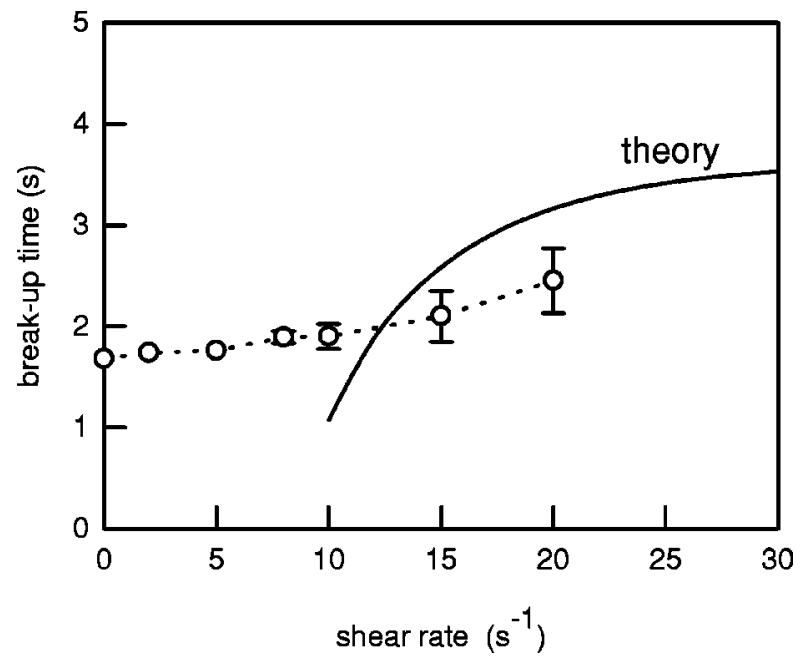

FIG. 9. Breakup time vs shear rate after a sudden decrease in shear rate (blend 4). The full line corresponds to the predictions of the Khakhar and Ottino theory.

with $n_{m}$ the refractive index of the matrix and $\lambda_{l}$ the wavelength of the light. A constant $\theta_{m}$ indicates that the periodicity of the growing interfacial disturbance does not change in time.

Figure 10 shows the evolution of the intensity distribution of the multistreak pattern at zero azimuthal angle after a sudden drop in shear rate. During shear the position of the intensity peak shifts to smaller angles with time. This evolution of the angle reflects an increase in the wavelength of the dominant disturbance [Eq. (13)]. With these data the evolution of the wavelength of the disturbance during flow can be assessed for the first time.

Figure 11(a) shows the dominant wavelength, normalized with respect to its initial value, for different stepdown experiments. In the range of shear rates covered here, the dominant wavelength evolves almost linearly with time. One would like to compare these

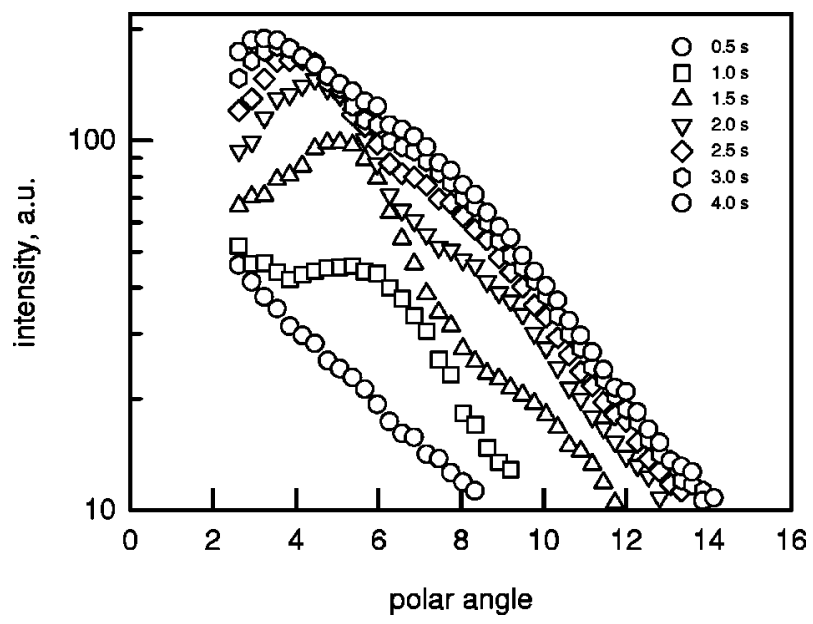

FIG. 10. Scattered light intensity vs scattering angle along $\phi=0^{\circ}$ for the SALS patterns of Fig. 7. 

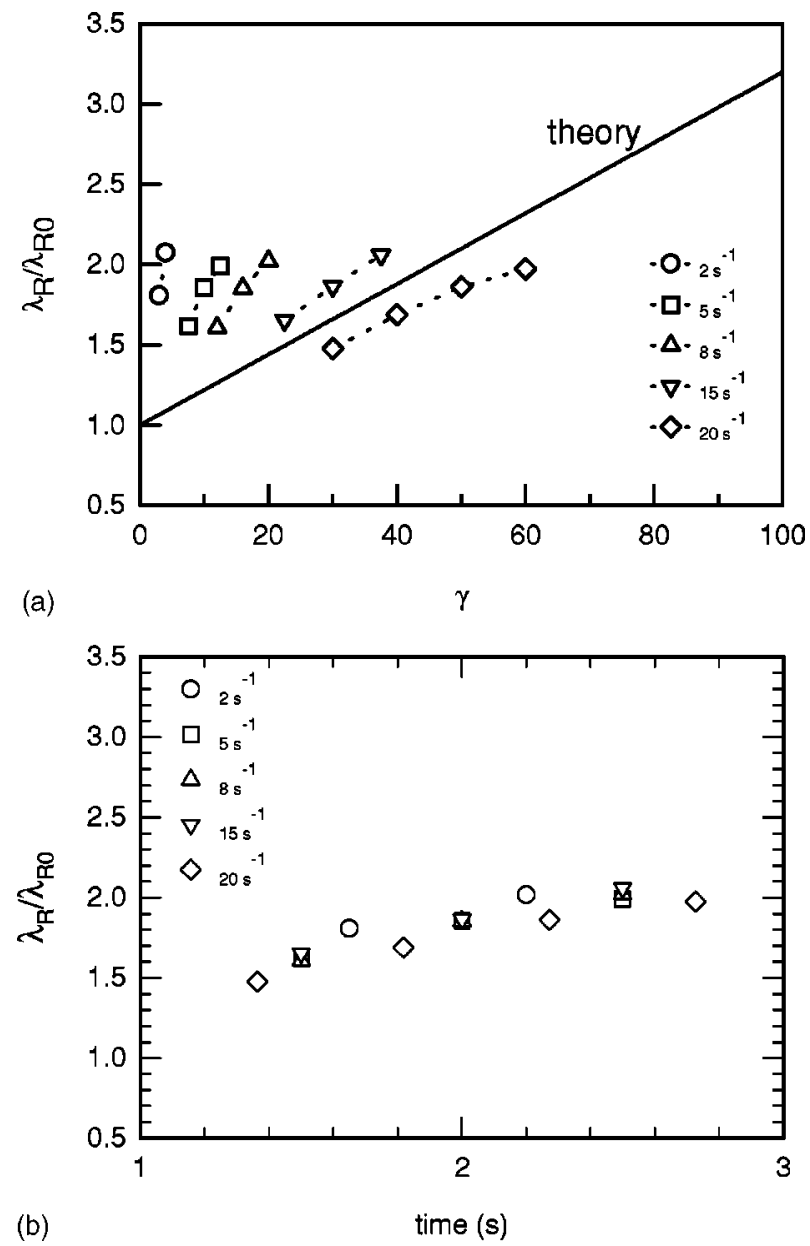

FIG. 11. (a) Wavelength of the surface instability vs strain after a sudden decrease in shear rate (blend 4). The full line represents the predictions of the Khakhar and Ottino theory. (b) Wavelength of the surface instability vs time after a sudden decrease in shear rate (blend 4).

data with the Khakhar and Ottino theory but this is not straightforward. In the model disturbances over a range of wavelengths simultaneously grow and decay [Khakhar and Ottino (1987)]. Hence the initial wavelength that produces the currently largest amplitude at any instant also changes with time. In the present experiments a substantial disturbance at a given dominant wavelength is already present on the interface of the fibrils at the moment the shear rate is reduced (see Fig. 7). Assuming that only this disturbance grows further in time, the Khakhar and Ottino theory predicts the evolution depicted as the full line in Fig. 11(a). It is observed that at higher shear rates the experimental results are in better agreement with the theoretical prediction. More importantly strain turns out not to be the controlling parameter. The present results actually seem to indicate that the dimensionless wavelength is time controlled rather than strain controlled, as illustrated in Fig. 11(b).

\section{CONCLUSIONS}

Filament breakup during shear flow has been investigated experimentally in dilute polymer blends by means of rheo-optical techniques, more specifically linear conserva- 
tive dichroism and SALS. With these techniques the breakup times can readily be determined after either increasing or decreasing the shear rate. In flows involving a sudden increase in shear rate the scaling suggested by the analysis of Khakhar and Ottino is satisfied quite well. In the theory the time elapsed between the onset of the surface instabilities and the moment of breakup is calculated. Is has been found here that the theoretical scaling relation can be applied to the total breakup time starting from the onset of flow, which is a more easily accessible entity. Introducing some elasticity in the droplet or matrix caused deviations from the scaling, as could be expected. With a viscoelastic droplet the scaling still holds when an effective interfacial tension is used. This seems to fail in the case of a viscoelastic matrix.

The breakup times and the wavelengths of the dominant disturbance have been measured on extended fibrils after a sudden drop in shear rate. The breakup times increase with shear rate as predicted by theory. The rate of growth of the dominating wavelength is found to be nearly independent of shear rate.

\section{ACKNOWLEDGMENTS}

This research has been partially funded by a GOA project (Project No. 98/06) from the Research Fund K. U. Leuven. P.V.P. is a Postdoctoral Fellow of the Fund for Scientific Research-Flanders (FWO-Vlaanderen).

\section{References}

Acrivos, A., "The breakup of small drops and bubbles in shear flows," Ann. N.Y. Acad. Sci. 404, 1-11 (1983).

Bentley, B. J. and L. G. Leal, "An experimental investigation of drop deformation and breakup in steady, two dimensional linear shear flows," J. Fluid Mech. 167, 241-283 (1986).

Elemans, P. H. M., H. L. Bos, J. M. H. Janssen, and H. E. H. Meijer, "Transient phenomena in dispersive mixing," Chem. Eng. Sci. 48, 247-276 (1993).

Fuller, G. G., Optical Rheometry of Complex Fluids (Oxford University Press, Oxford, 1995).

Grace, H. P., "Dispersion phenomena in high viscosity immiscible fluid systems and applications of static mixers as dispersion devices in such systems," Chem. Eng. Commun. 14, 225-277 (1982).

Grizzuti, N. and O. Bifulco, "Effects of coalescence and breakup on the steady state morphology of an immiscible blend in shear flow," Rheol. Acta 36, 406-415 (1997).

Janssen, J. M. H. and H. E. H. Meijer, "Droplet breakup mechanisms: Stepwise equilibrium versus transient dispersion,"' J. Rheol. 37, 597-608 (1993).

Khakhar, D. V. and J. M. Ottino, "Breakup of liquid threads in linear flows," Int. J. Multiphase Flow 13, 71-86 (1987).

Kuhn, W., "Spontane aufteilung von flussigkeitszylindern in Klein kugeln," Kolloid-Z. 132, 84-99 (1953).

Mewis, J., H. Yang, P. Van Puyvelde, P. Moldenaers, and L. M. Walker, "Small-angle light scattering study of droplet breakup in emulsions and polymer blends," Chem. Eng. Sci. 53, 2231-2239 (1998).

Mikami, T., R. G. Cox, and S. G. Mason, "Breakup of extending liquid threads," Int. J. Multiphase Flow 2, 113-138 (1975).

Minale, M., P. Moldenaers, and J. Mewis, "Effect of shear history on the morphology of immiscible polymer blends," Macromolecules 30, 5470-5475 (1997).

Rallison, J. M., "The deformation of small viscous drops and bubbles in shear flows," Annu. Rev. Fluid Mech. 16, 46-66 (1984).

Rumscheidt, F. D. and S. G. Mason, "Particle motions in sheared suspensions: XII Deformation and burst of fluid drops in shear and hyperbolic flow," J. Colloid Interface Sci. 16, 238-261 (1961).

Taylor, G. I., "The formation of emulsions in definable fields of flow," Proc. R. Soc. London, Ser. A 146, 501-523 (1934).

Tomotika, S., "On the instability of a cylindrical thread of a viscous liquid surrounded by another viscous fluid,' Proc. R. Soc. London, Ser. A 150, 322-337 (1935).

Tomotika, S., "Breaking of a drop of a viscous fluid immersed in another viscous fluid which is extending at a uniform rate,' Proc. R. Soc. London, Ser. A 153, 302-318 (1936).

Van Oene, H., "Modes of dispersion of viscoelastic fluids in flow," J. Colloid Interface Sci. 40, 448-467 (1972). 
Van Puyvelde, P., H. Yang, P. Moldenaers, and J. Mewis, "Rheo-optical study of relaxation phenomena in polymer blends," J. Colloid Interface Sci. 200, 86-94 (1998).

Vermant, J., P. Van Puyvelde, P. Moldenaers, J. Mewis, and G. G. Fuller, “Anisotropy and orientation of the microstructure in emulsions during shear flow," Langmuir 14, 1612-1617 (1998).

Vinckier, I., J. Mewis, and P. Moldenaers, "Stress relaxation as a microstructural probe for immiscible polymer blends," Rheol. Acta 36, 513-523 (1997).

Yang, H., H. Zhang, P. Moldenaers, and J. Mewis, "Rheo-optical investigation of immiscible polymer blends," Polymer 39, 5731-5737 (1998). 
